\title{
Effects of Fluoxetine on the Polysomnogram in Outpatients with Major Depression
}

\author{
Madhukar H. Trivedi, M.D., A. John Rush, M.D., Roseanne Armitage, Ph.D., \\ Christina M. Gullion, Ph.D., Bruce D. Grannemann, M.A., Paul J. Orsulak, Ph.D., \\ and Howard P. Roffwarg, M.D.
}

This study investigated the effects of open-label fluoxetine $(20 \mathrm{mg} / \mathrm{d})$ on the polysomnogram (PSG) in depressed outpatients $(n=58)$ who were treated for 5 weeks, after which dose escalation was available ( $\leqslant 40 \mathrm{mg} / \mathrm{d})$, based on clinical judgment. Thirty-six patients completed all 10 weeks of acute phase treatment and responded (HRS-D $\leqslant$ 10). PSG assessments were conducted and subjective sleep evaluations were gathered at baseline and at weeks 1, 5, and 10. Of the 36 subjects who completed the acute phase, 17 were reevaluated after 30 weeks on continuation phase treatment and 13 after approximately 7 weeks (range 6-8 weeks) following medication discontinuation. Acute phase treatment in responders was associated with significant increases in REM latency, Stage 1 sleep, and REM density, as well as significant decreases in sleep efficiency, total REM sleep, and Stage 2 sleep. Conversely, subjective measures of sleep indicated a steady improvement during acute phase treatment. After fluoxetine was discontinued, total REM sleep and sleep efficiency were found to be increased as compared to baseline.

[Neuropsychopharmacology 20:447-459, 1999] (C) 1999 American College of Neuropsychopharmacology. Published by Elsevier Science Inc.
KEY WORDS: Fluoxetine; REM sleep; Depression; Sleep EEG

Major depressive disorder is associated with several sleep EEG findings: (1) impaired sleep continuity (e.g., prolonged sleep latency, increased awakenings, reduced sleep efficiency); (2) enhanced rapid eye movement (REM) sleep (e.g., reduced REM latency, increased REM density, increased amount of REM in the first onehalf of the night); and (3) nonREM changes (e.g., decreased slow wave sleep, increased light, nonrestorative Stage 1 sleep) (for a review, see Reynolds and Kupfer 1987; Rush et al. 1991).

From the Department of Psychiatry (MHT, AJR, RA, CMG, BDG, PJO), University of Texas Southwestern Medical Center, Dallas, TX; and the Department of Psychiatry and Human Behavior (HPR), University of Mississippi Medical Center, Jackson, MS.

Address correspondence to: Madhukar H. Trivedi, M.D., Psychiatry at St. Paul POB 1, 5959 Harry Hines Blvd., Suite 520, Dallas, TX 75235. E-mail: mtrive@mednet.swmed.edu (214/648-4282; 648-4210, fax).

Received 4 September, 1996; revised 25 November, 1997; accepted 4 December, 1997.
Serotonin neurons play a key role in core behaviors of mood disorders (e.g., mood, sleep, sexual activity, appetite, circadian and seasonal rhythms, neuroendocrine functions, body temperature, motor activity, and cognitive function) (López-Ibor 1988; Meltzer and Lowy 1987; Rush et al. 1991). Serotonin affects the regulation of the sleep-wake cycle, the induction and maintenance of sleep, and the character of sleep-stage macroarchitecture (based on visual stage scores). The suprachiasmatic nucleus in the superior hypothalamus maintains the 24 hour sleep-wake cycle and synchronizes it with the environmental light cycle. The generation of nonREM sleep involves the basal forebrain, thalamus, hypothalamus, dorsal raphe nucleus, and nucleus tractus solitaris (Siegel 1990).

REM sleep expression is promoted in part by cholinergic neurons (Shiromani et al. 1988; Mitani et al. 1988) and inhibited by serotonergic and noradrenergic neurons in the dorsal pontine tegmentum (Luebke et al. 1992; Siegel 1990). The balance between monoaminergic 
and cholinergic influences appears to be critical for REM sleep (McCarley 1982).

Antidepressant medications produce characteristic changes in the sleep EEG of depressed patients, as well as normal controls. Whether the effects of antidepressant drugs on sleep of depressed patients differ from those in healthy controls is unclear because most data in normals are based on lower antidepressant doses used for shorter time periods than in depressed patients. Moreover, antidepressants affect neurotransmitters and their homeostatic balances which are potentially already affected in patients with depression.

Fluoxetine, a highly selective serotonin reuptake blocker, is as effective as other antidepressant medications in the treatment of major depressive disorder (Depression Guideline Panel 1993). In normal controls, fluoxetine increases REM latency, decreases REM sleep, reduces total sleep time and efficiency, and increases wakefulness and Stage 1 sleep. However, it is generally devoid of any effect on slow wave sleep (von Bardeleben et al. 1989; Saletu et al. 1991; Hendrickse et al. 1994; Nicholson and Pascoe 1988). In depressed patients, fluoxetine appears to prolong REM latency, reduce REM sleep, reduce REM density, and increase Stage 1 sleep (Hendrickse et al. 1994). However, Ciapparelli et al. (1992) found an absence of REM suppression after 4 weeks of treatment, although fluoxetine was associated with reduced slow wave sleep and sleep continuity, both of which appeared to persist even after 4 weeks of treatment with fluoxetine.

This study investigated the effects of fluoxetine on the polysomnogram (PSG) in depressed outpatients following 1,5 , and 10 weeks $\left(\mathrm{T}_{2}, \mathrm{~T}_{3}\right.$, and $\left.\mathrm{T}_{4}\right)$ of acute phase treatment with fluoxetine. It also provided descriptive information regarding longer term effects of fluoxetine on the PSG based on a subset of the original symptomatic patient group, once remitted, but still on continuation treatment (i.e., still taking fluoxetine), and on the same subset who successfully discontinued fluoxetine for at least 6 weeks following the continuation phase.

Based on the literature, we hypothesized that acute phase treatment with fluoxetine would (1) prolong REM latency; (2) decrease REM sleep; (3) prolong sleep latency; (4) increase Stage 1 sleep; (5) decrease Stages 2, 3 , and 4 sleep; and (6) increase awakenings in the first one-third of the night. We had no specific hypotheses regarding the timing of these effects or the effect of fluoxetine on sleep during or following continuation phase treatment.

\section{METHODS AND PROCEDURES}

\section{Subjects}

Subjects were selected from a pool of self-referred patients and symptomatically depressed volunteers recruited by local advertisements. Following an initial screening visit and written informed consent, potential subjects underwent full clinical evaluations including the Structured Clinical Interview for DSM-III-R (SCID) (Spitzer et al. 1992) administered by a trained clinical evaluator and confirmed by the first author. Depressive symptoms severity was measured by the 17-item Hamilton Rating Scale for Depression (HRS-D) (Hamilton 1960, 1967) and the 30-item Inventory for Depressive Symptomatology, both clinician-rated (IDS-C) and self-report (IDS-SR) versions (Rush et al. 1986, 1996).

All patients were required to meet DSM-III-R (APA, 1987) criteria for nonseasonal, nonpsychotic major depressive disorder, single or recurrent type, with moderate to severe symptomatology (as evidenced by a 17-item HRS-D score $\geqslant 16$ ) and be between $18-50$ years of age.

Patients with a history of any other psychiatric disorder (including psychoactive substance abuse within the previous 12 months) were excluded, as were patients who failed prior adequate treatment with fluoxetine (at least 4 weeks at $20 \mathrm{mg} /$ day). Individuals engaged in shift work within the last 6 months and those with independent sleep disorders (narcolepsy, apnea, bruxism, nyclonus) established either by history or by PSG evaluation were also excluded.

Only nonsteroidal, anti-inflammatory agents were permitted during the 2-week period preceding all PSG evaluations. All patients were completely drug-free for at least 2 weeks prior to the initial (baseline or $\mathrm{T}_{1}$ ) night of PSG recording. All patients were similarly drug-free except for the study medication (i.e., fluoxetine) and nonsteroidal antiinflammatory agents at all subsequent PSG evaluations $\left(T_{2}\right.$ to $\left.T_{5}\right)$, and they were completely drug-free at $\mathrm{T}_{6}$ (post-discontinuation).

\section{Acute Phase Treatment}

Patients received open-label fluoxetine throughout the study, initiated at a dose of $20 \mathrm{mg} /$ day (a.m. dosing). This dose was maintained for at least 5 consecutive weeks, at which time all subjects completed their third PSG assessment. Based on clinical judgment, the dose was increased to $40 \mathrm{mg}$ /day if satisfactory response had not occurred by week 5. Patients were evaluated weekly for symptom severity, side-effects, vital signs, and compliance for the first 10 weeks of treatment, and monthly for an additional 7 months. Symptom severity measures at each visit included the 17-item HRS-D and the 30-item IDS-C and IDS-SR. Treatment compliance was monitored by patient self-report, pill counts and fluoxetine and norfluoxetine blood levels.

Serum samples for fluoxetine and norfluoxetine were obtained in the morning approximately 24 hours after the last medication dose, before ingestion of medication for that day at every visit. Blood was drawn for analysis between 0800 and 1000 hours following the second 
night of PSG recording at week 1 and at each acute phase visit thereafter.

Fluoxetine and its metabolite, norfluoxetine, were isolated from serum by liquid-liquid extraction. They were then separated and quantified by reverse-phase, high-performance liquid chromatography (HPLC) with ultraviolet detection. Units reported are $\mathrm{ng} / \mathrm{ml}$. Withinrun precision was determined, yielding a coefficient of variation between $0.0 \%-5.1 \%$ for fluoxetine and $1.3 \%-$ $7.7 \%$ for norfluoxetine. The between-run coefficient of variation was $4.1 \%-6.8 \%$ for fluoxetine and $6.2 \%-8.8 \%$ for norfluoxetine (Orsulak et al. 1988).

\section{Continuation and Post-Continuation Treatment}

Patients who had achieved symptomatic remission at week $10($ HRSD $\leqslant 10)$ were continued on the same dose of fluoxetine through week 30 (i.e., an additional 20 weeks). At week $30\left(T_{5}\right)$, fluoxetine was discontinued and another PSG evaluation was performed. Patients were then followed for an additional 6-8 weeks drugfree at which time the final PSG evaluation occurred (week 37 or $\mathrm{T}_{6}$ ). During the continuation phase and following discontinuation, patients were evaluated monthly for symptom severity, side-effects, vital signs, and compliance. Thirty-eight patients completed $\mathrm{T}_{4}, 18$ completed $T_{5}$ and 13 completed $T_{6}$, and remained asymptomatic (HRS-D $\leqslant 10$ ). Paired $t$-tests were used to evaluate changes from $\mathrm{T}_{4}$ through $\mathrm{T}_{6}$.

\section{Polysomnographic Evaluations}

Baseline PSG assessments were conducted during two consecutive nights in the Department of Psychiatry Sleep Study Unit of the University of Texas Southwestern Medical Center on each measurement occasion. Regular sleep-wake habits were established in advance and confirmed by a self-reported 5-day sleep diary. Alcohol and napping were proscribed and caffeine restrictions were in place during the week of study. Each subject maintained individualized, regular bed and rise times for at least the 5 days prior to the PSG (as confirmed by home diary). An identical sleep-wake schedule was followed during the sleep laboratory studies.

The schedule for PSG assessments were $T_{1}$ (prior to beginning medication); $\mathrm{T}_{2}$ (following the first week on medication); $\mathrm{T}_{3}$ (following fifth week on medication); $\mathrm{T}_{4}$ (at the 10th week on medication); $\mathrm{T}_{5}$ (within \pm 7 days following the 30th week on medication) and $\mathrm{T}_{6}$ (within \pm 7 days of week 37 of the study or 7-8 weeks after medication discontinuation).

Electroencephalographic (EEG) data were collected from left (C3) and right (C4) central electrodes with a common ear reference passed through a $10-\mathrm{k}$ ohm resister to minimize nonhomogenous current flow (Nunez
1981). Monopolar, left and right electrooculograms, and bipolar chin-cheek electromyograms were also recorded. A full electrode montage, used on the first night in the sleep laboratory, included leg, chest, and abdomen leads, and a nasal-oral thermistor to rule out independent sleep disorders. Interelectrode impedance was maintained below 2-k ohms. All electrophysiologic signals were recorded on GRASS P-511 AC amplifiers and displayed on a paperless polygraph system designed and validated in-house. An amplifier sensitivity of 5 was used for EEG (50 uV, 0.5-second duration calibration), with half-amp low- and high-bandpass filters set at 0.3 and $30 \mathrm{~Hz}$. A $60-\mathrm{Hz}$ notch filter attenuated electrical noise. Interelectrode impedances were maintained below 2-k ohm. Amplifiers were calibrated before and after each night's sleep. As is standard procedure in our laboratory, EEG amplifiers were counterbalanced between hemispheres, across subjects, and between nights to rule out amplifier artifact as a contributing source to interhemispheric differences (Armitage et al. 1989) routinely evaluated in computer-analyzed EEG frequencies, but not reported here. Sleep EEG records were scored from C3 according to standard Rechtschaffen and Kales (1968) criteria by technicians trained at better than $90 \%$ agreement on an epoch-by-epoch basis.

\section{Polysomnographic Parameters}

A number of sleep variables were derived from the sleep-stage score data. Total time in bed (TIB) was computed as the total time from lights-out to wake-up time. Sleep onset latency was defined as the time elapsed before the first epoch of sleep in a 10-minute segment containing no more than 2 minutes of wakefulness or the time in minutes to the first half-minute of REM sleep, whichever was sooner (Rush et al. 1989). Total sleep period (TSP) was defined as the length of time from sleep onset to wake up. Total sleep time (TST) was defined as all sleep time in TIB. Sleep efficiency was computed as the ratio of all sleep time to total time in bed both including and excluding Stage 1 sleep. REM latency was equal to the number of minutes (including awake and movement (AMT) time) from sleep onset to the first epoch of REM sleep (irrespective of REM period duration). REM density was expressed as the average density per minute of REM evaluated on a $0-4$ scale. Minutes and percentages of each sleep stage and of AMT time were computed in relation to TSP. Minutes of AMT were also calculated by thirds of TSP. Arousals were defined as the total number of awake episodes of 0.5 minutes or longer duration in TSP.

The mean of 2 nights of PSG recording was used for all analyses. If an invalid sleep recording occurred on either of the first two nights, a third night of sleep measurement was conducted and the two most consistent of the three nights data were used. 


\section{Outcome Measures}

The 17-item HRS-D was the primary outcome measure. Response to acute phase treatment was defined as a 17item HRS-D score $\leqslant 10$ for one visit. An HRS-D score $\leqslant 10$ at each subsequent visit after week 10 was required for patients to continue in the protocol. The 30-item IDS-C and IDS-SR were used as secondary outcome measures.

To evaluate the subjective effects of fluoxetine on sleep, the sleep factors of the 17-item HRS-D and the 30item IDS-C and IDS-SR were used. The former rates early, middle, and late insomnia, each on a 0-2 scale. The IDS-C and IDS-SR both rate each of these three forms of insomnia on a 0-3 scale, as well as hypersomnia on a 0-3 scale. The total scores on these subscales (range $=0-6$ for the HRS-D and 0-12 for the IDS-C and IDS-SR, respectively) were computed on a weekly basis (acute phase) to gauge the particular sleep effects of the medication.

\section{Data Analyses}

Sixty-one subjects were enrolled in acute phase treatment, 3 of whom were subsequently excluded because of missing/incomplete baseline data, leaving a total eligible sample of 58 .

\section{Acute Phase Analysis Sample}

Twenty-two of the 58 subjects were not used in the acute phase analysis. Five of the 22 completed the acute phase and met the response criteria but did not complete all three sleep evaluations. One subject completed the acute phase but did not meet the response criteria. Sixteen subjects dropped out of the study before the end of the acute phase; 7 of which dropped prior to a response and 9 after a response but prior to the end of the acute phase. Of the 7 subjects who dropped out before a response, 3 did so after only 1 week of treatment and 4 after at least 4 weeks of treatment.

To evaluate changes in PSG parameters over all measurement occasions in acute phase treatment (i.e., baseline and weeks 1,5 , and 10), a repeated measures multivariate analysis of variance (MANOVA) was conducted using the 36 subjects with PSG measures at all 4 acute phase measurement occasions $\left(T_{1}\right.$ to $\left.T_{4}\right)$. Change in symptom severity, subjective sleep ratings and fluoxetine/norfluoxetine blood levels were evaluated during the acute phase.

\section{Follow-up Analysis Sample}

Of the 36 subjects included in the acute phase analysis, 14 dropped out before $T_{5}$ and 1 after $T_{5}$, with all visits meeting the response criteria. One subject relapsed before $T_{5}, 4$ relapsed at $T_{5}$ and 3 relapsed at $T_{6}$. To evaluate the long-term effects of fluoxetine in remitted depressed outpatients on mediation, we focused on the 13 subjects who completed $\mathrm{T}_{4}, \mathrm{~T}_{5}$, and $\mathrm{T}_{6}$, recognizing that these data were selective in that only those well enough and willing to complete all measurement occasions were included.

All parameters were examined for violation of symmetry and equal variances. Appropriate transformations were made prior to analyses. The natural logarithm was used for fluoxetine, norfluoxetine and total fluoxetine levels, as well as for REM latency, sleep latency, total minutes of AMT, and AMT by one-thirds of TSP. Squared transformation was used for sleep efficiency (including and excluding Stage 1), TIB, TSP, and TST. Ordinal transformations were used for minutes and \% Stages 3 and 4 sleep because no slow-wave sleep was evident in $36 \%$ of the PSG recordings. While we reported raw score values for ease of communication in the tables and figures, only transformed values were used in the statistical analyses which were divided into several parts.

1. Initially, repeated measures MANOVA were carried out for PSG parameters at baseline $\left(\mathrm{T}_{1}\right)$ through week $10\left(\mathrm{~T}_{4}\right)$, without controlling for blood levels on the 36 subjects who completed $\mathrm{T}_{4}$ and responded to treatment. This analysis tested the main hypotheses regarding the effect of fluoxetine on REM latency, \% REM, minutes of AMT in the first one-third of sleep, sleep latency, and sleep Stages 1-4. Similar analyses were then conducted to describe the effects of time on fluoxetine, norfluoxetine, and total blood levels, as well as on clinician-rated (HRS-D and IDS-C) and self-reported (IDS-SR) sleep factors.

Polynomial effects were tested when the multivariate main effect of time $(\mathrm{T})$ was significant. A significant linear component indicated a steady progressive increase or decrease in the parameter over the acute treatment period $\left(\mathrm{T}_{1}\right.$ to $\left.\mathrm{T}_{4}\right)$. A significant quadratic component indicated a single change in direction, such as an increase or decrease followed by no subsequent change in the opposite direction. The third possible and highest order component (with 4 measurement points) is a cubic component which indicates two change points or an S-shaped curve. One example would be a period of no change followed by a period of increase (or decrease), followed by a second period of no change. It should be noted that in instances in which more than one of the polynomial components was significant, the highest order component indicates the number of change points or general shape of the pattern of change. A second method used to understand the main effect was to test for specific differences between baseline $\left(T_{1}\right)$ and 
each of the other acute phase measurement occasions $\left(\mathrm{T}_{2}-\mathrm{T}_{4}\right)$ using a series of paired $t$-tests.

2. Following the same statistical procedures as described above, exploratory analyses included several important PSG measures not included in the original hypotheses: sleep efficiency (including and excluding Stage 1), total minutes AMT time, minutes of AMT in the second and third one-thirds of TSP, TST, and TIB.

3. To verify that the pattern of change in fluoxetine and norfluoxetine blood levels were consistent with the design of the prescribed dosing regimen, a series of analyses examining the pattern of change in fluoxetine, norfluoxetine and total blood level (the sum of the two) were conducted. Raw blood levels were transformed using a natural logarithm transformation to meet analyses requirements. The expected pattern of change was for a rapid increase between week 0 (baseline) and week 1 , followed by a slow increase (or steady level) between weeks 1 and 5, and a second period of increased levels between weeks 5 and 10 (reflecting an increase in oral dose for some patients). The analysis confirmed this expected pattern with all 3 measures (fluoxetine, norfluoxetine, and total blood level), showing a significant change in levels over time (fluoxetine: $F(3,28=1298.7, p<$ .0001; norfluoxetine: $F(3,28)=1956.4, p<.0001$; total blood level: $F(3,28)=4072.9, p<.0001)$. As expected, this change included significant linear, quadratic, and cubic components. Only the 31 subjects with complete blood level data were used in this analysis.

4. To explore the relationship between blood levels, HRS-D scores, and PSG measures, secondary analyses were conducted that included both the severity of depressive symptoms over time (HRS-D) and total blood levels (the log of total fluoxetine and norfluoxetine) as time-dependent covariates. This was carried out in a two-step process using repeated measures MANCOVAs with severity and drug level as the time-dependent covariates. The first step was used to test for homogeneity of slopes and included both measures as main effects and an interaction term. If no interaction was found, a second analysis was conducted using only the two measures (HRS-D score and blood level) as time-dependent covariates.

5. Analyses were conducted to explore the subjective experience of changes in sleep during acute phase treatment. Based on the sleep items from the HRS-D (items 4, 5, and 6), and the IDS-C and IDS-SR (items $1,2,3$, and 4), 3 measures of subjective sleep experience were constructed to explore these changes. The scoring for the IDS-SR measures were based on the Rush et al. (1996) analyses which indicated a reverse weighting on item 4 . A repeated measures MANOVA was used to test for an overall effect. Linear, qua- dratic, and cubic components, as well as t-tests, were used to describe the pattern of change. As these were exploratory analyses, probabilities should be used only to suggest possible effects.

6. A final series of paired $t$-tests were conducted on the 13 subjects who completed continuation treatment and the post-discontinuation follow-up. To determine how long-term exposure to fluoxetine may affect sleep, $t$-tests were used to compare the end of acute phase $\left(\mathrm{T}_{4}\right)$ and end of continuation phase $\left(\mathrm{T}_{5}\right)$ treatment. Paired $t$-tests were also conducted to explore possible differences between baseline $\left(\mathrm{T}_{1}\right)$ and $T_{5}$, and between $T_{1}$ and $T_{6}$ to determine the longterm effects of fluoxetine following 6-8 weeks of drug discontinuation in remitted depressed patients. Since these analyses were strictly exploratory, no Bonferroni corrections were used, and values are only presented as a guide for future research.

\section{RESULTS}

Table 1 gives the clinical and demographic characteristics for the total sample $(n=58)$, and for responders $(n=36)$. Table 2 provides the means and standard deviations of all sleep EEG measures at baseline $\left(\mathrm{T}_{1}\right)$ for the complete sample $(n=58)$. Using an unpaired $t$-test without Bonferroni correction, no differences were found between the 36 subjects whose data was used in the acute phase analysis and the 22 subjects whose data was not.

Only subjects with complete data at all 4 acute phase PSG measurement occasions [baseline $\left(T_{1}\right)$, week $1\left(T_{2}\right)$, week $5\left(\mathrm{~T}_{3}\right)$, and week $\left.10\left(\mathrm{~T}_{4}\right)\right]$ were included in the repeated measures analyses. Table 3 shows the PSG parameters for these 36 subjects at weeks $0,1,5$, and 10 . While Table 3 shows raw scores (i.e., nontransformed), data were transformed for analytical purposes (see above). All $p$ values below are a test of the overall multivariate $F$ of change over time, and the significant linear, quadratic, and cubic components of the effect are noted in Table 3.

Consistent with our hypotheses, fluoxetine significantly altered REM sleep in that REM latency increased throughout acute phase treatment $(F(3,33)=59.5, p<$ .0001). However, this effect included a marked immediate increase followed by a slight decline after the first week on medication (Figure 1) and it remained higher than baseline at all 3 acute phase measurement occasions (weeks 1,5 , and 10). Percent REM sleep was significantly reduced $(F(3,33)=22.8, p<.0001)$ from baseline on all occasions. There was a sharp decline at week 1 and then subsequent small increases from week 1 to week 5 and then week 10 (Figure 2).

The hypotheses regarding changes in sleep continuity early in the night were generally confirmed. Minutes 
Table 1. Baseline Features of the Full Sample and the Analyzed Sample

\begin{tabular}{|c|c|c|c|c|c|c|}
\hline \multirow[b]{2}{*}{ Measure } & \multicolumn{3}{|c|}{ Total Sample $(n=58)$} & \multicolumn{3}{|c|}{ Analyzed Sample $(n=36)$} \\
\hline & $n$ & Range & $\begin{array}{l}\text { Mean } \pm \text { SD } \\
\quad \text { or } \%\end{array}$ & $n$ & Range & $\begin{array}{l}\text { Mean } \pm \text { SD } \\
\text { or } \%\end{array}$ \\
\hline Age (yr) & 58 & $(18-50)$ & $34.6 \pm 8.8$ & 36 & $(18-50)$ & $36.5 \pm 9.6$ \\
\hline Education (yr) & 58 & $(9-24)$ & $14.0 \pm 2.2$ & 36 & $(11-24)$ & $14.3 \pm 2.4$ \\
\hline Female & 38 & - & $65.5 \%$ & 24 & - & $66.7 \%$ \\
\hline Male & 20 & - & $34.5 \%$ & 12 & - & $33.3 \%$ \\
\hline \multicolumn{7}{|l|}{ Axis $\mathrm{V}$} \\
\hline Current level & 57 & $(41-70)$ & $55.5 \pm 4.9$ & 36 & $(45-70)$ & $55.7 \pm 4.7$ \\
\hline Highest level & 57 & $(5-80)$ & $63.2 \pm 10.1$ & 36 & $(5-80)$ & $62.8 \pm 11.8$ \\
\hline Age at onset (yr) & 55 & $(7-49)$ & $24.8 \pm 11.1$ & 35 & $(8-49)$ & $26.2 \pm 12.1$ \\
\hline Number of episodes & $49^{\mathrm{a}}$ & $(1-6)$ & $2.0 \pm 1.3$ & $30^{a}$ & $(1-4)$ & $1.8 \pm 1.0$ \\
\hline Length of illness (yr) & 55 & $(0-38)$ & $10.0 \pm 9.5$ & 35 & $(0-38)$ & $10.1 \pm 10.5$ \\
\hline \multicolumn{7}{|l|}{ Length of current episode } \\
\hline (months) & 57 & $(1-168)$ & $35.3 \pm 36.1$ & 36 & $(1-168)$ & $37.4 \pm 38.0$ \\
\hline \multicolumn{7}{|l|}{ Depressive symptom severity } \\
\hline HRS-D & 58 & $(16-32)$ & $21.8 \pm 3.3$ & 36 & $(16-29)$ & $21.2 \pm 2.7$ \\
\hline IDS-C & 58 & $(27-58)$ & $39.2 \pm 8.0$ & 36 & $(27-58)$ & $38.1 \pm 8.0$ \\
\hline IDS-SR & 54 & $(21-68)$ & $43.4 \pm 10.0$ & 33 & $(28-61)$ & $42.3 \pm 8.4$ \\
\hline \multicolumn{7}{|l|}{ Course of illness } \\
\hline Recurrent, complete recovery & 16 & - & $28.1 \%$ & 8 & - & $22.2 \%$ \\
\hline Recurrent, incomplete recovery & 13 & - & $22.8 \%$ & 8 & - & $22.2 \%$ \\
\hline Single episode & 22 & - & $38.6 \%$ & 16 & - & $44.4 \%$ \\
\hline Unknown & 7 & - & $12.1 \%$ & 4 & - & $11.1 \%$ \\
\hline \multicolumn{7}{|l|}{ Depressive subtype (MDD Only) } \\
\hline RDC Primary & 47 & - & $81.0 \%$ & 30 & - & $83.3 \%$ \\
\hline RDC Secondary & 7 & - & $12.1 \%$ & 4 & - & $11.1 \%$ \\
\hline Unknown & 1 & - & $1.7 \%$ & 2 & - & $5.6 \%$ \\
\hline RDC Endogenous & 26 & - & $44.8 \%$ & 16 & - & $44.4 \%$ \\
\hline RDC Nonendogenous & 31 & - & $53.5 \%$ & 19 & - & $52.8 \%$ \\
\hline Unknown & 1 & - & $1.7 \%$ & 1 & - & $2.8 \%$ \\
\hline DSM-IV Melancholic & 11 & - & $19.0 \%$ & 8 & - & $22.2 \%$ \\
\hline DSM-IV Nonmelancholic & 47 & - & $81.0 \%$ & 28 & - & $77.8 \%$ \\
\hline Nonpsychotic & 58 & - & $100.0 \%$ & 36 & - & $100.0 \%$ \\
\hline \multicolumn{7}{|l|}{ Family history subtype } \\
\hline Depression Spectrum Disease & 21 & - & $36.2 \%$ & 12 & - & $33.3 \%$ \\
\hline Familial Pure Depressive Disease & 9 & - & $15.5 \%$ & 5 & - & $13.9 \%$ \\
\hline Sporadic Depressive Disorder & 10 & - & $17.2 \%$ & 6 & - & $16.7 \%$ \\
\hline Familial Bipolar Disease & 1 & - & $1.7 \%$ & 1 & - & $2.8 \%$ \\
\hline Unknown & 2 & - & $3.5 \%$ & 12 & - & $33.3 \%$ \\
\hline
\end{tabular}

${ }^{a}$ Number of episodes too many to count.

of AMT in the first one-third of TSP increased significantly $(F(3,33)=3.4, p<.03)$ over acute phase treatment, although values at weeks 1,5 , and 10 (taken as individual measurement occasions) were not higher than baseline. Sleep latency tended to decrease $(F(3,33)=$ 2.5, $p<.08)$ at weeks 1 and 10, but was no different from baseline at week 5 .

The predictions of increased Stage 1 and decreased Stage 2 sleep were also confirmed. Both minutes $(F(3,33)=$ $9.9, p<.0001)$ and $\%(F(3,33)=12.6, p<.0001)$ Stage 1 significantly increased. These effects were characterized by a rapid rise in Stage 1 sleep between baseline and week 1 , which slowed between weeks 1 and 5 , and remained constant between weeks 5 and 10. Minutes of Stage 2 sleep rose initially, and then decreased $(F(3,33)=$ $9.1, p<.0002)$. The changes from baseline revealed only a significant difference at week 10 , with minutes of Stage 2 lower than at baseline. Similar results were obtained for $\%$ Stage $2(F(3,33)=8.9, p<.0002)$.

The hypothesized changes in sleep Stages 3 and 4 were not found. Minutes and \% Stages 3 and 4 did not significantly change from baseline (Wilcoxon signed ranks test).

The follow-up, time-dependent covariate analyses revealed an interaction between HRS-D score and total blood level for REM latency $[F(1,99)=7.2, p<.002]$, \% $\operatorname{REM}(F(1,99)=12.9, p<.0005]$, minutes of Stage 2 $(F(1,99)=11.1, p<.002]$ and \% Stage $2(F(1,99)=6.7$, $p<.02]$. The presence of interactions between the two covariates and PSG measures indicates that the effects of HRS-D score and blood level are not independent of PSG measures over the duration of acute phase treat- 
Table 2. PSG Parameters at Baseline $\left(\mathrm{T}_{1}\right)(n=58)$

\begin{tabular}{lccc}
\hline Parameter & Mean \pm SD & Parameter & Mean \pm SD \\
\hline TIB (min) & $444.1 \pm 52.5$ & Stage 1 min in TSP & $70.0 \pm 29.3$ \\
TSP (min) & $422.5 \pm 54.4$ & \% Stage 1 in TSP & $16.5 \pm 6.5$ \\
TST (min) & $393.7 \pm 50.6$ & Stage 2 min in TSP & $225.8 \pm 43.2$ \\
REM latency (min) & $81.3 \pm 25.7$ & \% Stage 2 in TSP & $53.5 \pm 8.9$ \\
\% Stage REM in TSP & $17.1 \pm 4.3$ & Stages 3 and 4 min in TSP & $12.4 \pm 14.9$ \\
REM density & $2.4 \pm 0.6$ & $\%$ Stages 3 and 4 min in TSP & $3.0 \pm 4.0$ \\
AMT in TSP (min) & $29.2 \pm 17.2$ & \% Sleep efficiency & $88.7 \pm 4.7$ \\
AMT in first 1/3 TSP (min) & $7.0 \pm 5.3$ & \% Sleep efficiency (minus stage 1) & $73.0 \pm 8.9$ \\
AMT in second 1/3 TSP (min) & $11.3 \pm 11.6$ & Sleep latency (min) & $18.6 \pm 9.8$ \\
AMT in third 1/3 TSP (min) & $11.1 \pm 9.0$ & & \\
\hline
\end{tabular}

ment. To assist with the interpretation of this interaction, subjects were divided into three groups based on changes in total fluoxetine/norfluoxetine blood levels between treatment weeks 5 and 10 . The first group consisted of subjects whose blood level either remained unchanged or showed a slight decrease. The second group included subjects whose blood levels showed a slight increase. The third group included subjects whose blood levels showed a marked increase from week 5 to week 10. Of note is that this group consisted of subjects who also had an increase in fluoxetine dose from $20 \mathrm{mg} /$ day to $40 \mathrm{mg} /$ day due to nonresponse. Figure 3 shows the three groups defined by changes in blood levels.

The pattern of change in REM latency is similar across the first two groups. There was an initial increase at week 1 , followed by a gradual decrease over the remaining 9 weeks. For the third group, the interaction was the result of an increased blood level (due to increased dose), while HRS-D score decreased between weeks 5 and 10 . The interactions for \% REM are similar, with the pattern of change reversed (i.e., initial decrease followed by recovery for the first two groups, whereas the third group showed a further decrease between weeks 5 and 10).

The interaction found for Stage 2 sleep was not caused by differences in changes during treatment but rather by differences at the beginning of treatment. Subjects in the third group start well above the first and second groups for Stage 2 sleep at week $0\left(\mathrm{~T}_{1}\right)$ and show no initial change in the amount of Stage 2 sleep. In contrast, subjects in the first two groups showed an increase in Stage 2 sleep at week 1. Interestingly, at week 5 all three groups are at baseline levels (i.e., the first two groups show decreases which followed the initial in-

Table 3. PSG Measures at All Acute Phase Sleep Occasions for Acute Phase Responders $(n=36)^{a}$

\begin{tabular}{|c|c|c|c|c|}
\hline & $\begin{array}{c}\mathrm{T}_{1} \\
\text { Mean } \pm \mathrm{SD}\end{array}$ & $\begin{array}{c}T_{2} \\
\text { Mean } \pm S D\end{array}$ & $\begin{array}{c}\mathrm{T}_{3} \\
\text { Mean } \pm \mathrm{SD}\end{array}$ & $\begin{array}{c}\mathrm{T}_{4} \\
\text { Mean } \pm \mathrm{SD}\end{array}$ \\
\hline TIB (min) & $442.1 \pm 57.6$ & $437.3 \pm 53.9$ & $442.1 \pm 56.5$ & $436.4 \pm 55.7$ \\
\hline TST within TSP & $389.8 \pm 56.4$ & $389.1 \pm 47.0$ & $389.1 \pm 50.9$ & $384.0 \pm 52.0$ \\
\hline Time from sleep onset to wake-up (min) & $420.3 \pm 59.9$ & $420.9 \pm 48.7$ & $424.0 \pm 53.5$ & $419.5 \pm 54.5$ \\
\hline REM latency (min) (includes AMT) (LQC) & $80.1 \pm 25.1$ & $156.2 \pm 69.5$ & $141.6 \pm 59.5$ & $133.2 \pm 54.8$ \\
\hline$\%$ stage REM in TSP (QL) & $16.9 \pm 4.4$ & $10.8 \pm 4.5$ & $12.6 \pm 4.5$ & $14.5 \pm 4.5$ \\
\hline Average REM density / REM min in TSP (L) & $2.4 \pm 0.6$ & $2.6 \pm 0.8$ & $2.9 \pm 0.8$ & $3.1 \pm 0.9$ \\
\hline AMT in TSP ( $\mathrm{min})$ & $31.0 \pm 18.5$ & $32.3 \pm 20.9$ & $35.4 \pm 19.6$ & $36.0 \pm 21.9$ \\
\hline AMT in first $1 / 3$ TSP (min) (L) & $6.4 \pm 5.0$ & $5.7 \pm 3.9$ & $8.1 \pm 5.4$ & $8.5 \pm 7.3$ \\
\hline AMT in second 1/3 TSP (min) & $12.5 \pm 13.3$ & $14.1 \pm 15.9$ & $14.4 \pm 13.4$ & $13.6 \pm 10.9$ \\
\hline AMT in third $1 / 3$ TSP (min) & $12.1 \pm 9.7$ & $12.5 \pm 10.0$ & $13.3 \pm 9.0$ & $13.9 \pm 10.5$ \\
\hline Stage 1 min in TSP (LQC) & $69.3 \pm 27.6$ & $87.9 \pm 34.5$ & $102.3 \pm 41.9$ & $105.4 \pm 43.9$ \\
\hline$\%$ Stage 1 in TSP (LQC) & $16.4 \pm 6.3$ & $21.0 \pm 8.4$ & $23.9 \pm 8.6$ & $24.7 \pm 8.7$ \\
\hline Stage 2 min in TSP (LQ) & $222.9 \pm 48.7$ & $233.3 \pm 48.0$ & $216.7 \pm 41.0$ & $201.4 \pm 47.0$ \\
\hline$\%$ Stage 2 in TSP (LC) & $53.1 \pm 9.9$ & $55.5 \pm 9.8$ & $51.5 \pm 8.8$ & $48.4 \pm 10.9$ \\
\hline Stages 3 and $4 \mathrm{~min}$ in TSP & $12.7 \pm 15.5$ & $10.5 \pm 13.1$ & $8.0 \pm 10.0$ & $7.8 \pm 10.0$ \\
\hline$\%$ Stages 3 and 4 iin TSP & $3.2 \pm 4.4$ & $2.5 \pm 3.3$ & $1.9 \pm 2.4$ & $1.9 \pm 2.6$ \\
\hline$\%$ sleep efficiency & $88.2 \pm 5.2$ & $89.2 \pm 5.7$ & $88.1 \pm 5.1$ & $88.1 \pm 6.5$ \\
\hline \% sleep efficiency (minus stage 1) (LQ) & $72.5 \pm 9.0$ & $68.9 \pm 9.7$ & $65.1 \pm 9.8$ & $64.3 \pm 10.5$ \\
\hline Sleep latency $(\mathrm{min})(8 / 10: 1)$ & $17.9 \pm 10.2$ & $15.1 \pm 10.5$ & $15.3 \pm 9.1$ & $15.7 \pm 13.4$ \\
\hline
\end{tabular}

${ }^{a} \mathrm{~T}_{1}=$ baseline; $\mathrm{T}_{2}=1$ week on drug; $\mathrm{T}_{3}=5$ weeks on drug; $\mathrm{T}_{4}=10$ weeks on drug.

Numbers in boldface indicate significant changes from baseline $\left(\mathrm{T}_{1}\right)(p<.05)$. LQC indicates a significant $(p<.05)$ linear $(\mathrm{L})$, quadratic $(\mathrm{Q})$, or cubic (C) component. 


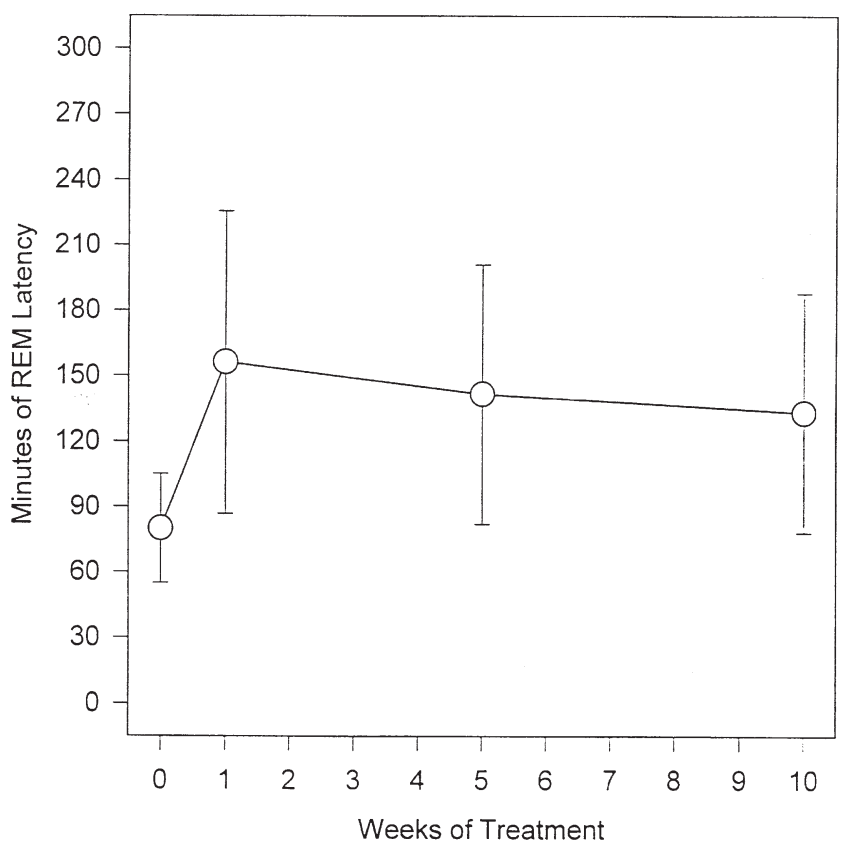

Figure 1. The effects of fluoxetine on REM latency.

crease, while the third group remained unchanged). Finally, by week 10, the first and third groups fall below baseline levels for Stage 2 sleep, yet the second group remained unchanged.

No significant interactions were found for minutes AMT in the first one-third of the night, sleep latency, Stage 1 and \% Stage 1 . Thus, results are reported only for the main effects model. For AMT in the first one-third of the night, only the HRS-D covariate was significant $(F(1,100)=6.4, p<.007]$, indicating an increase in AMT in the first one-third of the night as HRS-D score decreased during treatment. For sleep latency, only total blood level was a significant predictor $[F(1,100)=6.4$, $p<.02$ ], indicating a decrease in sleep latency as blood level increases. Both HRS-D score $[F(1,00)=4.9, p<$ $.03]$ and total blood level $[F(1,100)=10.2, p<.002]$ predicted minutes in Stage 1 sleep, but in opposite directions. Stage 1 increased as HRS-D score decreased, whereas Stage 1 decreased as total blood level increased. For $\%$ Stage 1 , the same pattern was found for total blood level $[F(1,100)=14.5, p<0.002]$, but the HRS-D covariate effect was only marginal $[F(1,100)=2.8, p<.10]$.

For exploratory analyses of PSG measures for which no hypotheses were made, only REM density significantly increased $(F(3,33)=17.0, p<.0001)$, and sleep efficiency (minus Stage 1$)$ significantly decreased $(F(3,33)=$ $12.6, p<.0004$ ) over time. None of the other PSG measures changed significantly.

A parallel set of repeated measures MANOVA analyses were conducted using only the final night of PSG recording to allow for comparisons of our results for means of 2 nights to single night PSG recordings. Three

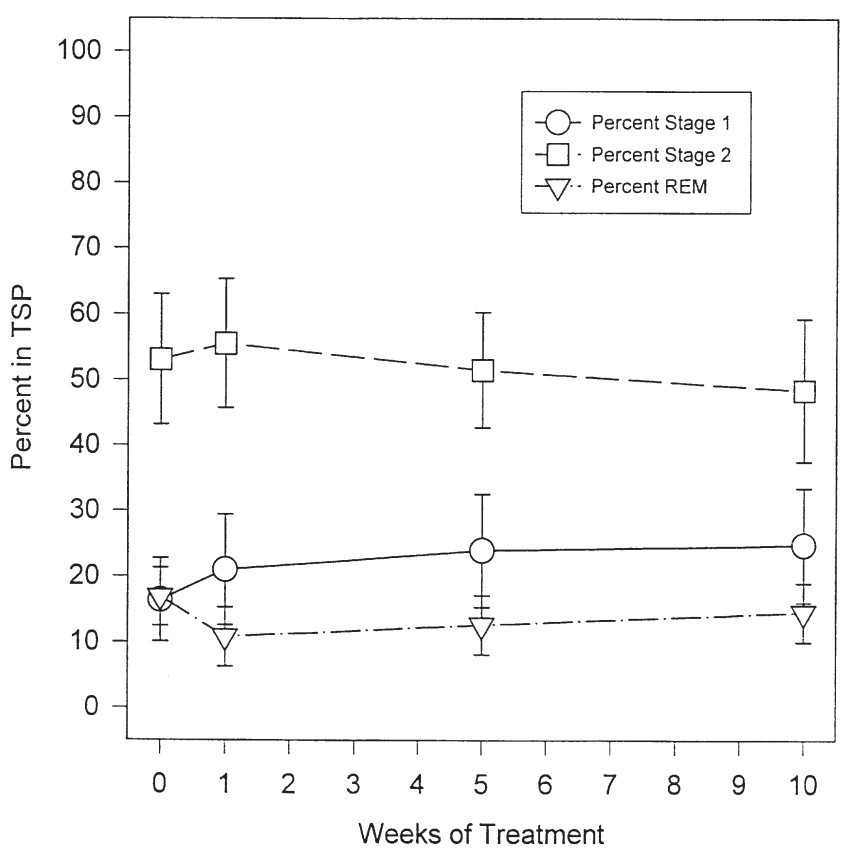

Figure 2. The effects of fluoxetine on \% Stage 1, \% Stage 2, and \% REM sleep.

subjects had only 1 night of PSG recording at the end of acute phase treatment $\left(\mathrm{T}_{4}\right)$ and they were not included in this analysis. All measures were transformed as described above.

The results of the final night analyses were comparable to the average of two nights with no differences in overall significance. The only significant differences found were in the presence (or absence) of quadratic (AMT time in the first one-third of the night) or cubic (sleep efficiency) components.

The quadratic component for Stage 2 and the cubic component for \% Stage 2 were not significant in the final night analyses. Similarly, the cubic component was now only marginally significant for Stage 1 sleep and was nonsignificant for \% Stage 1.

All 3 subjective measures of insomnia showed a significant change over acute phase treatment (HRS-D: $F(3,33)=33.8, p<.001$; IDS-C: $F(3,33)=29.8, p<.001$; IDS-SR: $F(3,32)=15.9, p<.0001)$. The overall pattern was a steady decrease in the amount of reported insomnia. The $t$-tests indicated that the improvement began at week 1 and continued throughout acute phase treatment (see Table 4).

\section{Study Completers}

To inform future studies, a second set of descriptive data were compiled (Table 4) and exploratory analyses conducted on the transformed data of the 13 subjects who completed $\mathrm{T}_{4}, \mathrm{~T}_{5}$, and $\mathrm{T}_{6}$. These 13 were then compared to the 23 subjects who completed only acute 


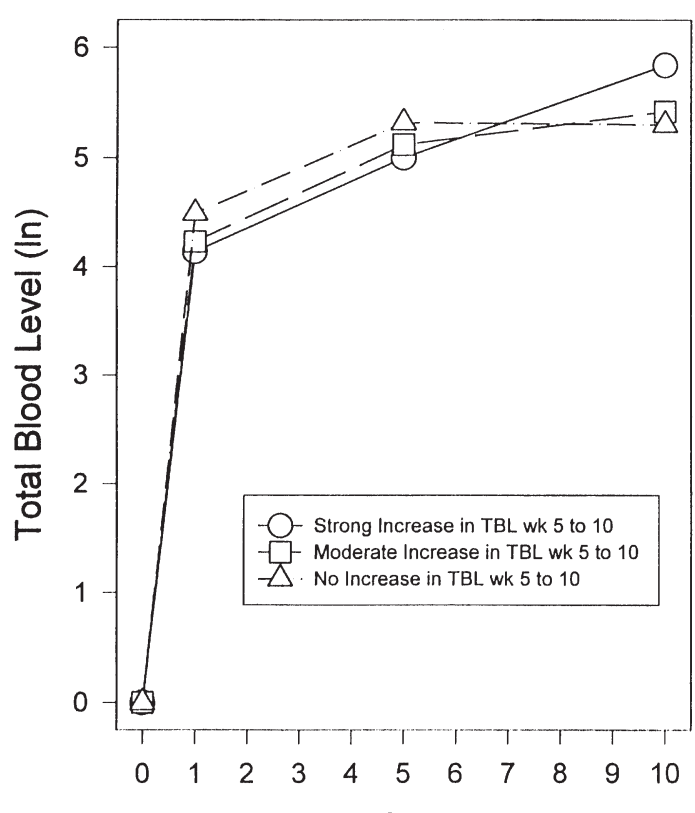

A

Weeks of Treatment

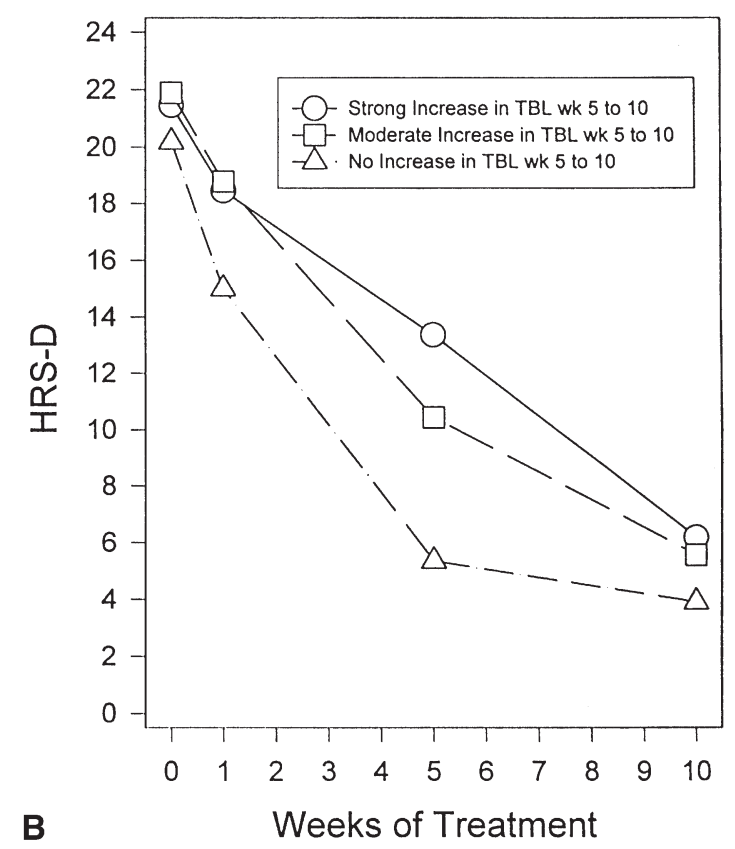

Figure 3. Three groups defined by blood level change: (A) total blood level (lv); (B) HRS-D.

phase treatment to identify any obvious differences between the two groups. The groups did not differ with regard to blood levels at $\mathrm{T}_{4}$ or PSG parameters. However, at week 10 , the 13 completers had significantly lower HRS-D total scores $(t(34)=2.3, p<.04)$ (study completers: mean $=4.0 \pm 2.2$; acute phase only completers: mean $=6.0 \pm 2.7$ ).

Table 4 shows the PSG parameters for the 13 subjects who completed $\mathrm{T}_{4}, \mathrm{~T}_{5}$, and $\mathrm{T}_{6}$. Comparison of subjects after 10 weeks on fluoxetine with themselves after 30 weeks ( \pm 4 days) on drug (i.e., end of continuation phase treatment or $T_{5}$ ), revealed only a significant increase in $\%$ REM. To determine how much change from baseline persisted after 30 weeks of treatment (i.e., comparable to the drug-free but symptomatically depressed state), comparisons between $T_{1}$ (baseline) and $T_{5}$ were conducted. These analyses revealed that Stage 1 (minutes and \%), REM density, sleep efficiency (minus Stage 1) and REM latency were still elevated as compared to baseline (Table 4).

Once drug-free for at least 7 weeks ( \pm 5 days) (i.e., at $\mathrm{T}_{6}$ or post-discontinuation), the following differences were found between $T_{4}$ and $T_{6}$ and between $T_{5}$ and $T_{6}$. For both comparisons, minutes and \% Stage 1 were lower at $\mathrm{T}_{6}$; Stage 2 (both $\%$ and minutes) was higher at $\mathrm{T}_{6}$; AMT time was lower at $\mathrm{T}_{6}$; and both sleep efficiency and sleep efficiency (minus Stage 1 ) were lower at $\mathrm{T}_{6}$. Furthermore, \% REM was higher, REM density was lower and minutes of AMT in the first, second and third one-thirds of the night were lower at $T_{6}$ than at $T_{5}$ (paired $t$-tests).
Of note is the apparent "recovery" of minutes of Stages 1 and 2, sleep efficiency (minus Stage 1), and REM density at $T_{6}$ to approximately pretreatment (i.e., baseline) levels. On the other hand, both REM latency and AMT time in the first one-third of the TSP showed only significant decreases between the end of acute treatment $\left(\mathrm{T}_{4}\right)$ and the drug-free post-treatment followup $\left(\mathrm{T}_{6}\right)$. For the subjective sleep measures, all $\left(\mathrm{T}_{4}, \mathrm{~T}_{5}\right.$ and $\mathrm{T}_{6}$ ) were lower than baseline $\left(\mathrm{T}_{1}\right)$ and did not differ from each other (see Table 5). Since all of these findings are based on a very small, highly select sample using post hoc analyses, they should be viewed with caution.

\section{DISCUSSION}

The major findings of this study were that symptomatic response to acute phase treatment with fluoxetine lightened sleep, in that it increased Stage 1 and decreased Stage 2. REM latency was also longer, total REM sleep decreased, and REM density increased. A weak increase in AMT time in the first one-third of the night, and a trend toward a decrease rather than the predicted increase in sleep latency were also found. No changes in Stages 3 and 4 sleep was found. Most effects were apparent by the first week on fluoxetine and largely persisted throughout the 10 weeks of acute phase treatment.

When symptom severity (HRS-D score) and blood level change were used to predict acute phase treatment related PSG response patterns, the interaction of both accounted for the pattern of change in REM latency, \% 
Table 4. PSG Parameters for Subjects Completing the 30-Week Treatment Trial and 7 Weeks of Follow-up $(n=13)^{d}$

\begin{tabular}{|c|c|c|c|c|}
\hline & $\begin{array}{c}\mathrm{T}_{1} \\
\text { Mean } \pm \mathrm{SD}\end{array}$ & $\begin{array}{c}\mathrm{T}_{4} \\
\text { Mean } \pm \mathrm{SD}\end{array}$ & $\begin{array}{c}T_{5}{ }^{e} \\
\text { Mean } \pm \text { SD }\end{array}$ & $\begin{array}{c}\mathrm{T}_{6} \\
\text { Mean } \pm \mathrm{SD}\end{array}$ \\
\hline TIB (min) & $428.4 \pm 51.9$ & $424.3 \pm 49.7$ & $418.4 \pm 77.8$ & $418.4 \pm 58.9$ \\
\hline TST within TSP & $374.9 \pm 53.6$ & $372.0 \pm 48.5$ & $366.9 \pm 64.2$ & $384.4 \pm 62.8$ \\
\hline $\begin{array}{l}\text { Time from sleep onset } \\
\text { to wake-up (min) }\end{array}$ & $404.5 \pm 57.6$ & $409.5 \pm 49.4$ & $398.5 \pm 73.8$ & $405.0 \pm 63.4$ \\
\hline REM latency (min) & & & & \\
\hline (includes AMT) & $76.3 \pm 22.0$ & $121.3 \pm 43.8$ & $104.3 \pm 34.9$ & $66.7 \pm 15.0^{b, c}$ \\
\hline \% Stage REM in TSP & $16.2 \pm 4.3$ & $14.9 \pm 4.3$ & $18.7 \pm 3.1^{\mathrm{a}}$ & $19.0 \pm 5.2^{b}$ \\
\hline Average REM Density/ & & & & \\
\hline REM min in TSP & $2.3 \pm 0.4$ & $3.0 \pm 0.7$ & $2.9 \pm 0.7$ & $2.4 \pm 0.5^{b, c}$ \\
\hline AMT in TSP (min) & $30.2 \pm 16.1$ & $38.0 \pm 13.9$ & $32.1 \pm 13.7$ & $21.0 \pm 11.8^{b}$ \\
\hline AMT in first $1 / 3 \mathrm{TSP}(\mathrm{min})$ & $7.5 \pm 7.1$ & $8.1 \pm 3.7$ & $7.2 \pm 3.7$ & $5.8 \pm 3.2^{b}$ \\
\hline AMT in second $1 / 3$ TSP & $13.5 \pm 11.7$ & $12.3 \pm 3.9$ & $13.0 \pm 7.7$ & $7.4 \pm 4.8^{b}$ \\
\hline AMT in third $1 / 3$ TSP & $9.2 \pm 7.0$ & $17.8 \pm 13.7$ & $11.9 \pm 4.9$ & $8.0 \pm 4.2^{b}$ \\
\hline Stage $1 \mathrm{~min}$ in TSP & $71.7 \pm 33.8$ & $107.2 \pm 45.2$ & $100.4 \pm 36.5$ & $71.6 \pm 20.6^{b, c}$ \\
\hline$\%$ Stage 1 in TSP & $17.7 \pm 8.1$ & $25.9 \pm 9.4$ & $24.7 \pm 6.8$ & $17.9 \pm 5.0^{b, c}$ \\
\hline Stage $2 \min$ in TSP & $208.0 \pm 51.8$ & $186.2 \pm 48.8$ & $175.6 \pm 40.6$ & $209.7 \pm 49.3^{c}$ \\
\hline$\%$ Stage 2 in TSP & $51.0 \pm 10.2$ & $45.9 \pm 10.4$ & $44.3 \pm 7.9$ & $51.4 \pm 9.1^{c}$ \\
\hline Stages 3 and $4 \mathrm{~min}$ in TSP & $13.8 \pm 18.1$ & $8.6 \pm 11.6$ & $8.6 \pm 11.3$ & $11.9 \pm 14.5$ \\
\hline$\%$ Stages 3 and 4 in TSP & $3.9 \pm 5.9$ & $2.1 \pm 3.0$ & $2.2 \pm 3.2$ & $3.2 \pm 4.3$ \\
\hline \% sleep efficiency & $87.4 \pm 6.0$ & $87.6 \pm 3.5$ & $87.9 \pm 3.9$ & $91.6 \pm 4.7^{b, c}$ \\
\hline $\begin{array}{l}\text { \% sleep efficiency } \\
\text { (minus stage } 1 \text { ) }\end{array}$ & $70.6=$ & & & $744+7 \Omega^{b, c}$ \\
\hline Sleep latency $(\mathrm{min})(8 / 10: 1)$ & $15.7 \pm 8.8$ & $13.1 \pm 6.6$ & $16.8 \pm 15.0$ & $10.7 \pm 5.4$ \\
\hline
\end{tabular}

${ }^{a}$ Significant change between $\mathrm{T}_{4}$ and $\mathrm{T}_{5}(p<.05)$.

${ }^{b}$ Significant change between $\mathrm{T}_{4}$ and $\mathrm{T}_{6}(p<.05)$.

'Significant change between $\mathrm{T}_{5}$ and $\mathrm{T}_{6}(p<.05)$.

${ }^{d} \mathrm{~T}_{1}=$ baseline; $\mathrm{T}_{4}=10$ weeks on drug; $\mathrm{T}_{5}=30$ weeks on drug; $\mathrm{T}_{6}=7$ weeks off drug.

${ }^{e} n=12$ (one patient had no $T_{5}$ sleep).

Number in boldface indicates significant change between that time period and baseline $\left(T_{1}\right)$

REM and Stage 2 sleep. Conversely, for Stage 1 sleep, both severity and blood levels independently contributed to the response pattern. Finally, only blood level change accounted for sleep latency changes whereas, only symptom severity accounted for the pattern of change in AMT in the first one-third of the night.
The increase in REM latency, coupled with a decrease in total REM sleep, confirms the findings from our pilot study (Hendrickse et al. 1994) and is similar to other reports for most antidepressant medications, including tricyclics and selective serotonin reuptake inhibitors (SSRIs) (Heiligenstein et al. 1994; Keck et al.

Table 5. Symptom Severity, Sleep Factors, and Blood Levels for Subjects Completing the 30-Week Trial and the 7-Week Follow-up $(n=13)$

\begin{tabular}{lcccr}
\hline & $\begin{array}{c}\mathbf{T}_{\mathbf{1}} \\
\text { Mean } \pm \mathbf{S D}\end{array}$ & $\begin{array}{c}\mathbf{T}_{\mathbf{2}} \\
\text { Mean } \pm \mathbf{S D}\end{array}$ & $\begin{array}{c}\mathbf{T}_{\mathbf{3}} \\
\text { Mean } \pm \mathbf{S D}\end{array}$ & $\begin{array}{c}\mathbf{T}_{\mathbf{4}} \\
\text { Mean } \pm \mathbf{S D}\end{array}$ \\
\hline $\begin{array}{l}\text { Symptom severity } \\
\text { HRS-D }\end{array}$ & & & & \\
IDS-C & $20.6 \pm 3.1$ & $4.0 \pm 2.2$ & $2.4 \pm 1.9$ & $2.4 \pm 2.3$ \\
$\quad$ IDS-SR & $32.5 \pm 7.0$ & $6.6 \pm 4.2$ & $5.6 \pm 6.1$ & $4.5 \pm 3.2$ \\
Sleep factors & $32.3 \pm 8.0$ & $10.0 \pm 5.9$ & $9.8 \pm 10.8$ & $10.6 \pm 6.2$ \\
$\quad$ HRS-D & $3.5 \pm 1.5$ & $\mathbf{0 . 9} \pm \mathbf{1 . 4}$ & $\mathbf{0 . 2} \pm \mathbf{0 . 4}$ & $\mathbf{0 . 9} \pm \mathbf{1 . 3}$ \\
IDS-C & $4.5 \pm 1.7$ & $\mathbf{1 . 2} \pm \mathbf{1 . 7}$ & $\mathbf{0 . 3} \pm \mathbf{0 . 6}$ & $\mathbf{1 . 1} \pm \mathbf{1 . 6}$ \\
IDS-SR & $3.6 \pm 1.5$ & $\mathbf{1 . 5} \pm \mathbf{1 . 1}$ & $\mathbf{1 . 8} \pm \mathbf{2 . 2}$ & $\mathbf{2 . 0} \pm \mathbf{1 . 6}$ \\
Blood levels & & & & \\
$\quad$ Fluoxetine & $0.0 \pm 0.0$ & $121.7 \pm 63.1$ & $118.6 \pm 9.45$ & $0.0 \pm 0.0$ \\
$\quad$ Norfluoxetine & $0.0 \pm 0.0$ & $150.1 \pm 57.7$ & $139.7 \pm 69.2$ & $0.0 \pm 0.0$ \\
\hline
\end{tabular}

${ }^{a}$ Blood levels for $\mathrm{T}_{1}(n=13), \mathrm{T}_{4}(n=12)$, and $\mathrm{T}_{5}$ and $\mathrm{T}_{6}(n=10)$.

Numbers in boldface indicate significant change from baseline $\left(\mathrm{T}_{1}\right)$ for subjective sleep factors $(p<.05$, paired $t$-test). 
1991; Kupfer et al. 1981; van Bemmel et al. 1993, Vogel et al. 1990). The significant increase in REM density may be purely secondary to increased oculomotor activity induced by fluoxetine (Armitage et al. 1995; Schenck et al. 1992), although its direct effect (i.e., blockade of serotonin reuptake) is clearly expected to increase 5-HT availability at all post-synaptic 5-HT receptors (especially 5- $\mathrm{HT}_{1 \mathrm{C}}$ ) and thus decrease REM sleep. Moreover, since REM density is based on average REM minutes, it reflects an actual increase in the number of eye movements on fluoxetine, despite decreased REM time.

The findings of increased Stage 1, decreased Stage 2, and increased AMT time in the first one-third of the night also replicate earlier findings, suggesting an overall lightening of sleep secondary to fluoxetine (Hendrickse et al. 1994; Keck et al. 1991). In their sample of 16 subjects treated with citalopram (an SSRI), van Bemmel et al. (1993) found increased Stages 1 and 2 sleep, but did not find significant changes in sleep continuity variables. One possible explanation for the discrepancy in sleep continuity findings is that they analyzed only total time awake as opposed to the first, second, and third one-thirds of the night, whereas Stage 2 sleep may be increased secondary to citalopram's histaminergic effect.

Interestingly, in spite of the above-mentioned lightening of sleep with sleep continuity disruptions, subjective reports of insomnia-related disturbances showed significant improvement throughout acute phase treatment. However, the insomnia items of the HRS-D and IDS may not be sensitive to all dimensions of subjective sleep disturbance. When specifically queried about sleep quality and the number and duration of awakenings, $37.5 \%$ of women and $12 \%$ of men have reported more disturbed sleep while on fluoxetine (Armitage et al. 1995). Moreover, insomnia secondary to fluoxetine has been reported by others (Dorsey et al. 1996). However, the current findings do not concur and may be related to the fact that this investigation was a sleep study of responders only. It is possible that patients with insomnia secondary to fluoxetine drop out early in the course of the treatment. Nevertheless, subjective sleep measures may not always show the same magnitude and direction of change as do objective sleep parameters. Finally, sleep latency continues to improve with symptomatic improvement while on fluoxetine.

In the subset of patients who continued on fluoxetine for 30 weeks and were studied again after 7-8 weeks off medication, there was evidence of some accommodation to the acute phase effects. However, while still on medication and once fluoxetine was discontinued, the short REM latency, exceptionally short sleep latency, and increased REM all suggest REM rebound.

From a theoretical perspective, drug-induced improvement of depression has been suggested to be mediated by changes in sleep physiology. Vogel et al. (1990) and Kupfer et al. (1981) have argued that REM sleep suppression appears to be a correlate of the mechanism of action of antidepressant medications. Our findings of increased REM latency are comparable to the results of earlier findings of the effects of SSRIs. Additionally, some nonpharmacological approaches, such as total or partial REM deprivation, have been shown to ameliorate depressive symptoms. In contrast, however, some recent studies with bupropion (Nofzinger et al. 1995) and nefazondone (Armitage et al. 1994) appear to point to a different mechanism of action for at least some antidepressant medications. Thus, while most antidepressants suppress REM sleep, this effect may not be required for antidepressant efficacy for at least some medications. Of note is that with citalopram, the amount of initial and sustained REM suppression was unrelated to clinical change (van Bemmel et al. 1993). Thus, even with REM suppressing medications, efficacy may be unrelated to REM sleep effects.

This study has several limitations: (1) a fairly high rate of dropout during the long-term treatment with fluoxetine leading to the need for cautious interpretation of the results for $T_{5}$ and $T_{6}$ PSG parameters; (2) the age range was restricted (18-50 years) and included $65.5 \%$ females, thus precluding definitive analyses of differential age or gender effects; and (3) the current report does not address the differences between responders and nonresponders since the study design specifically included only those patients who had achieved full symptomatic remission at week 10 .

In summary, acute phase response to fluoxetine is associated with lightening of sleep, REM suppression, and prolonged REM latency, but is not associated with changes in Stages 3 and 4 sleep. However, these physiological changes are not uniformly mirrored by subjective sleep reports. Descriptive data suggest that further physiological adaptations may occur after several months of continuation phase treatment. Studies of the comparative effects on sleep of the newer antidepressant medications (e.g., nefazodone, paroxetine, sertraline and venlafaxine) are clearly indicated.

\section{ACKNOWLEDGMENTS}

The authors wish to express their appreciation to John R. Debus, M.D., Jeff Bostic, M.D., Terry Cook, L.V.N., and David Dunnam, B.S., for assistance with data collection; Darwynn Cole, and the technical staff of the Sleep Study Unit; to Fastword Inc., of Dallas, David Savage and Theresa Ranne, for their secretarial assistance; and to Kenneth Z. Altshuler, M.D., Stanton Sharp Distinguished Chair, Professor and Chairman, for his administrative support. This study was supported in part by a contract from Eli Lilly and Company and a grant from the National Alliance for Research in Schizophrenia and Depression (Dr. Trivedi). 


\section{REFERENCES}

American Psychiatric Association (1987): Diagnostic and Statistical Manual of Mental Disorders, 3rd ed., revised. Washington, DC, American Psychiatric Press

Armitage R, Hoffmann R, Loewy D, Moffitt A (1989): Variations in period-analyzed EEG asymmetry in REM and NREM sleep. Psychophysiology 26:329-336

Armitage R, Rush AJ, Trivedi M, Cain J, Roffwarg HP (1994): The effects of nefazodone on sleep architecture in depression. Neuropsychopharmacology 10:123-127

Armitage R, Trivedi M, Rush AJ (1995): Fluoxetine and oculomotor activity during sleep in depressed patients. Neuropsychopharmacology 12:159-165

Ciapparelli A, Dani A, Figura A, Gemignani A, Guazzelli M (1992): The effects of fluoxetine (FL) on sleep pattern: A clinical and all-night EEG study in patients with major depression. J Sleep Res 1:41

Depression Guideline Panel (1993): Clinical Practice Guideline. Depression in Primary Care: Volume 2: Treatment of Major Depression. (AHCPR Publication No. 93-0551.) Rockville, MD, U.S. Dept. of Health and Human Services, Public Health Service, Agency for Health Care Policy and Research.

Dorsey CM, Lukas SE, Cunningham SL (1996): Fluoxetineinduced sleep disturbance in depressed patients. Neuropsychopharmacology 14:437-442

Hamilton M (1960): A rating scale for depression. J Neurol Neurosurg Psychiatry 23:56-62

Hamilton M (1967): Development of a rating scale for primary depressive illness. Br J Soc Clin Psychol 6:278-296

Heiligenstein JH, Faries DE, Rush AJ, Andersen JS, Pande AC, Roffwarg HP, Dunner D, Gillin JC, James SP, Lahmeyer H, Zajecka J, Tollefson GD, Gardner DM (1994): Latency to rapid eye movement sleep as a predictor of treatment response to fluoxetine and placebo in nonpsychotic depressed outpatients. Psychiatry Res 52:327-339

Hendrickse WA, Roffwarg HP, Granneman BD, Orsulak PJ, Armitage R, Cain JW, Battaglia J, Debus J, Rush AJ (1994): The effects of fluoxetine on the polysomnogram of depressed outpatients: A pilot study. Neuropsychopharmacology 10:85-91

López-Ibor JJ Jr. (1988): The involvement of serotonin in psychiatric disorders and behavior. $\mathrm{Br} \mathrm{J}$ Psychiatry 153(suppl 3):26-39

Luebke JI, Greene RW, Semba K, Kamondi A, McCarley RW, Reinder PB (1992): Serotonin hyperpolarizes cholinergic low-threshold burst neurons in the rat laterodorsal tegmental nucleus in vitro. Proc Natl Acad Sci USA 89:743-747

Keck PE Jr, Hudson JI, Dorsey CM, Campbell PI (1991): Effect of fluoxetine on sleep. Biol Psychiatry 29:618-619

Kupfer DJ, Spiker DG, Coble PA, Neil JF, Ulrich R, Shaw DH (1981): Sleep and treatment prediction in endogenous depression. Am J Psychiatry 138:429-434

McCarley RW (1982): REM sleep and depression: Common neurobiological control mechanisms. Am J Psychiatry 139:565-570

Meltzer HY, Lowy MT (1987): The serotonin hypothesis of depression. In Meltzer HY (ed), Psychopharmacology:
The Third Generation of Progress. New York, Raven Press, pp 513-526

Mitani A, Ito K, Hallanger AE, Wainer BH, Kataoka K, McCarley RW (1988): Cholinergic projections from the laterodorsal and pedunculopontine tegmental nuclei to the pontine giganticellular tegmental field in the cat. Brain Res 451:397-402

Nicholson AN, Pascoe PA (1988): Studies on the modulation of the sleep-wakefulness continuum in man by fluoxetine, a 5-HT uptake inhibitor. Neuropharmacology 27:597-602

Nofzinger EA, Reynolds III CF, Thase ME, Frank E, Jennings JR, Fasiczka AL, Sullivan LR, Kupfer DJ (1995): REM sleep enhancement by bupropion in depressed men. Am J Psychiatry 152:274-276

Nunez P (1981): Electrical Fields of the Brain. New York, Oxford Universities Press

Orsulak PJ, Kenney JT, Debus JR, Crowley G, Wittman PD (1988): Determination of the antidepressant fluoxetine and its metabolite in serum by reverse-phase HPLC, with ultraviolet detection. Clin Chem 34:1875-1878

Rechtschaffen A, Kales A (eds) (1968): A Manual of Standardized Terminology, Techniques and Scoring System for Sleep Stages of Human Subjects. Washington, DC, National Institute of Health, (NIH Publ No. 204). US Government Printing Office

Reynolds CF III, Kupfer DJ (1987): Sleep research in affective illness. State-of-the-art circa 1987. Sleep 10:199-215

Rush AJ, Cain JW, Raese J, Stewart RS, Waller DA, Debus JR (1991): Neurobiological bases for psychiatric disorders. In Rosenberg RN (ed), Comprehensive Neurology. New York, Raven Press, pp 555-608

Rush AJ, Giles DE, Jarrett RJ, Fedlman-Koffler F, Debus JR, Weissenberger JE, Orsulak PJ, Roffwarg HP (1989): Reduced REM latency predicts response to tricyclic medication in depressed outpatients. Biol Psychiatry 26:61-72

Rush AJ, Giles DE, Schlesser MA, Fulton CL, Weissenburger JE, Burns CT (1986): The Inventory for Depressive Symptomatology (IDS). Psychiatry Res 18:65-87

Rush AJ, Gullion CM, Basco MR, Jarrett RB, Trivedi MH (1996): The inventory of depressive symptomatology (IDS): Psychometric properties. Psychol Med 26:477-486

Saletu B, Frey R, Krupka M, Anderer P, Grunberger J, See WR (1991): Sleep laboratory studies on the single-dose effects of serotonin reuptake inhibitors paroxetine and fluoxetine on human sleep and awakening qualities. Sleep 14:439-447

Schenck CS, Mahowald MW, Kim SW, O'Connor KA, Hurwitz TD (1992): Prominent eye movements during NREM sleep and REM sleep behavior disorder associated with fluoxetine treatment of depression and obsessive-compulsive disorder. Sleep 15:226-235

Shiromani PJ, Armstrong DM, Berkowitz A, Jeste DV, Gillin JC (1988): Distribution of choline acetyltransferase immunoreacive somata in the feline brainstem: Implications for REM sleep generation. Sleep 11:1-16

Siegel JM (1990): Mechanisms of sleep control. J Clin Neurophysiol 7:49-65

Spitzer RL, Williams JBW, Gibbon M, First MB (1992): The Structured Clinical Interview for DSM-III-R (SCID) I: 
History, rationale, and description. Arch Gen Psychiatry 49:624-629

van Bemmel AL, van den Hoofdakker RH, Beersma DGM, Bouhuys AL (1993): Changes in sleep polygraphic variables and clinical state in depressed patients during treatment with citalopram. Psychopharmacology 113: 225-230 von Bardeleben U, Steiger A, Gerken A, Holsboer F (1989): Effects of fluoxetine upon pharmacoendocrine and sleep-EEG parameters in normal controls. Int Clin Psychopharmacol 4(Suppl 1):1-5

Vogel GW, Buffenstein A, Minter K, Hennessey A (1990): Drug effects on REM sleep and on endogenous depression. Neurosci Biobehav Rev 14:49-63 